\title{
Effects of Two Different Modes of Task Practice during Lower Limb Constraint-Induced Movement Therapy in People with Stroke: A Randomized Clinical Trial
}

\author{
Auwal Abdullahi $\mathbb{D}^{1,2}$ Naima Umar Aliyu $\mathbb{D}^{3},{ }^{3}$ Ushotanefe Useh $\mathbb{D}^{4},{ }^{4}$ \\ Muhammad Aliyu Abba $\mathbb{D}^{1},{ }^{1}$ Mukadas Oyeniran Akindele $\mathbb{D}^{1,},{ }^{1,4}$ Steven Truijen $\left(\mathbb{D},{ }^{2}\right.$ \\ and Wim Saeys $\mathbb{( 1 )}^{2}$ \\ ${ }^{1}$ Department of Physiotherapy, Bayero, University Kano, PMB 3011, Gwarzo Road, Kano, Nigeria \\ ${ }^{2}$ Department of Physiotherapy and Rehabilitation Sciences, University of Antwerp, Antwerp, D.R.312, 2610 Wilrijk, Belgium \\ ${ }^{3}$ Department of Physiotherapy, Muhammad Abdullahi Wase Teaching Hospital, Kano, Off Audu Bako Way, Nassarawa G.R.A, \\ $P M B, 3160$ Kano, Nigeria \\ ${ }^{4}$ Lifestyle Diseases Research Entity, Faculty of Health Sciences, North-West University, South Africa
}

Correspondence should be addressed to Auwal Abdullahi; aabdullahi.pth@buk.edu.ng

Received 24 October 2020; Revised 16 December 2020; Accepted 19 January 2021; Published 1 February 2021

Academic Editor: Gabriela Delevati Colpo

\begin{abstract}
Copyright (c) 2021 Auwal Abdullahi et al. This is an open access article distributed under the Creative Commons Attribution License, which permits unrestricted use, distribution, and reproduction in any medium, provided the original work is properly cited.
\end{abstract}

Background. Constraint-induced movement therapy (CIMT) is used for the rehabilitation of motor function after stroke. Objectives. The aim of this study was to compare the effects of lower limb CIMT that uses number of repetition of tasks with the one that uses number of hours of practice. Method. The study was a randomized clinical trial approved by the Ethics Committee of Kano State Ministry of Health. Fifty-eight people with stroke participated in the study. Groups 1 and 2 performed daily 600 repetitions and 3 hours of task practice, respectively, 5 times weekly for 4 weeks. Motor impairment (primary outcome), balance, functional mobility, knee extensor spasticity, walking speed and endurance, and exertion before and after commencement of activities were assessed at baseline and postintervention. The data was analyzed using Friedmann and MannWhitney $U$ tests. Result. The results showed that there was only significant difference $(p<0.05)$ in knee extensor spasticity (group 1 (median $=0(0)$, mean rank $=27.50)$; group $2($ median $=0(0)$, mean rank $=31.64)$ ), exertion before commencement of activities (group 1 (median $=0(0.5)$, mean rank $=21.90)$; group $2($ median $=1(0.5)$, mean rank $=37.64)$ ), and exertion after commencement of activities (group 1 (median=1(1), mean rank=20.07); group 2 (median =1(0), mean rank=39.61) postintervention in favour of the experimental group (group 1)). Conclusion. The group 1 protocol is more effective at improving outcomes after stroke.

\section{Introduction}

One of the significant activities of daily living (ADL) human beings carry out is mobility such as walking. The ability to walk is largely controlled by the central nervous system (CNS) in which the brain is an integral part [1-3]. This control is however impaired in conditions that affect the brain such as stroke in which motor function of the lower limbs is impaired $[4,5]$. Thus, one of the most important goals in stroke rehabilitation is to help patients regain motor function of the lower limbs and consequently the ability to walk independently as much as possible in order to ensure the ability to carry out ADLs [6]. To improve walking ability after stroke, constraint-induced movement therapy (CIMT) is used $[7,8]$.

The CIMT is a movement rehabilitation technique used to improve motor function after stroke. It was initially invented to force the use of the deafferented forelimbs in 
monkeys [9], but this has since then been translated to improve motor function following stroke in humans [1013]. The most important components of CIMT for both upper and lower limbs are mass task practice with the affected limb, constraint of the unaffected limb, and transfer package [7, 11-19]. CIMT has a very strong evidence base. It helps improve walking ability, walking speed and other gait parameters, movement quantity and quality, and neurophysiological functions of the brain $[7,18,20-22]$. It also increases the expression of Growth-Associated Protein 43 (GAP-43) and numbers of $\Delta$ FosB-positive cells which are some of the biomarkers that play important roles in neural cell proliferation and neural and synaptic plasticity [20]. Similarly, movement of the lower limbs is very essential to brain health since reduced mobility results in a decrease in neural stem cell proliferation capability and altered cell cycle [23]. The neural stem cells are the self-renewing and multipotent cells that generate neurons and glial cells in the embryonic and adult brains [24].

One of the problems with CIMT is the prescription of the appropriate intensity of task practice enough to induce neuroplastic changes and recovery of motor function. This is because traditionally in both upper and lower limb CIMT protocols, duration in hours of practice is used as the measure of intensity of practice. In the lower limb CIMT specifically, the duration for practice has been reported to range between a couple of minutes to six hours per day $[8,23-$ 26]. A CIMT protocol that uses 6 hours of task practice is the original CIMT, whereas the one that uses a shorter duration is known as the modified CIMT. However, the CIMT protocol that uses duration of practice has been argued to be not straight forward as duration does not correspond to a specific number of repetitions [27]. In contrast, how many times tasks are practiced is an important driver of recovery of motor function. Thankfully, the number of repetitions required for this recovery has been reported both in animals and humans, and it ranges between 289 and 1000 repetitions per day [28-35]. In particular, the studies on upper limb showed that using number of repetitions of task practice as a measure of intensity is feasible and effective at improving outcomes such as motor function, real-world arm use, and upper limb self-efficacy [3234]. Unfortunately, majority of CIMT protocols used hours of practice as the measure of intensity of tasks being practiced. When hours are used, it is difficult to know whether patients achieved the amount of practice required for recovery. Therefore, it is wiser to ask our patients to carry out specific number of task repetitions during CIMT instead of leaving this open ended. This will help them to carry out the required number of repetitions known to result in recovery. The aim of this study was to compare the effects of a lower limb CIMT protocol using number of repetitions and the one using number of hours of practice. The study hypothesis was as follows: there will be a significant difference between CIMT protocol using number of repetitions and the one using number of hours of practice. This is because the protocol using number of repetitions seems to be more efficient, specific, and easy to follow in upper limb CIMT $[27,36]$.

\section{Materials and Method}

2.1. Study Design. The study was a randomized clinical trial approved by the Research Ethics Committee of the Kano State Ministry of Health (MOH/Off/799/T.I/688). It was also registered with the Pan African Clinical Trial Registry (PACTR201806003363142). In addition, consent for participation in the study and the publication of the study results was obtained from the participants.

2.2. Sample Size Estimate. The sample size of the study was estimated using G Power software version 3.1 [37]. The parameters used to estimate the sample size were effect size $=0.4$, power $=0.8$, and alpha value $=0.05$. However, since 52 is the minimum sample size required to obtain a significant effect, 6 (10\% attrition rate) was added to make it 58 . This sample size calculation was based on the primary outcome which is motor function measured using lower limbs Fugl Meyer. In addition, a moderate effect size (0.4) was used in order to have a larger sample size even though a previous study by Danlami and Abdullahi has obtained a large effect size of $>0.6[38]$.

2.3. Participants. The study participants were stroke patients. The inclusion criteria used were as follows: participants with hemorrhagic or ischemic stroke in any stage of stroke, who were $\geq 18$ years old, with good cognitive ability (a score of $\geq 24$ on minimental state examination), with asymmetrical stance (assessed by observing the participant's standing posture), with ability to stand and walk with minimal assistance assessed using functional ambulation category, and who have $\geq 15$ degrees of active knee flexion in the affected limb in standing position. This is because people with stroke have an extensor synergy pattern in the lower limbs which could impair their ability to flex the knee [39]. However, participants with severe pain that could interfere with training and those with hemineglect indicated by a cutoff of $<44$ on the star cancellation test were excluded from the study [40]. The study settings were physiotherapy departments of Murtala Muhammad Specialists Hospital and Muhammad Abdullahi Teaching Hospital in Kano, Nigeria.

The recruitment of the participants was done consecutively by a trained therapist in each of the study sites. A simple random technique using sealed opaque envelopes was used to allocate the participants into groups 1 and 2 . The period for recruitment and follow-up was between $16^{\text {th }}$ May 2018 and $4^{\text {th }}$ July 2018.

2.4. Intervention and Control. Participants in both groups performed the following tasks: stepping forward, backward stepping, side stepping, ball kicking, and stair climbing. However, group 1 performed each of the tasks 40 times per session (altogether 200 repetitions), three sessions (morning, afternoon, and evening) per day (altogether 600 repetitions), five days per week, and with constraint applied only during practice sessions for four consecutive weeks. 600 repetitions were used because results of previous studies showed that for motor recovery to be achieved, task repetitions in the range of 300 to 800 per day is required $[28,29,32]$. On the other hand, group 2 performed modified CIMT consisting 
of three hours of task practice per day, five days per week, and with constraint applied during practice sessions for four consecutive weeks. Participants and their caregivers in both groups were trained on the first day by a very well-trained therapist in each of the study centers who was blinded to the aim of the study on how to carry out the tasks, and they were then asked to perform the tasks, two times a week at home under the supervision of the trained caregivers. This means that the five days a week treatment periods were divided into two days a week whereby the participants carried out the tasks at home under the supervision of their caregivers and three days a week whereby the participants carry out the tasks in the clinic under the supervision of the trained therapist. However, participants in both groups did not receive any other therapy such as conventional therapy throughout the study period.

To constrain the unaffected limb during practice, participants were told not to use the unaffected extremity during the practice. This was achieved by asking the participants to keep the limb still with the foot flat at one point and the hip and knee in full extension. In addition, participants were blinded to what each one was doing by requesting that they do not discuss the treatment given to them with other participants to avoid contamination bias. Furthermore, from time to time, calls were put through to the patients' relatives to monitor how the patients comply with the intervention's protocols. A logbook was also used by the relatives to record compliance with the protocols. When compliance is $>90 \%$, it indicates high adherence with the protocol [41]. Participants were also seen three times in the clinic, and this also assisted with the monitoring of the compliance.

2.5. Study Outcomes. In this study, lower limb motor function was the primary outcome which was measured using lower limb Fugl Meyer (FM). The secondary outcomes were balance measured using the Berg balance scale (BBS), functional mobility measured using the Rivermead Mobility Index (RMI), knee extensor spasticity assessed using the modified Ashworth scale (MAS), walking speed measured using the Ten-Meter Walk Test (10MWT), and endurance measured using the Six-Minute Walk Test (6MWT). In addition, exertion before and after commencement of activities was measured using hard activity chart for rating perceived exertion [42]. The lower limb Fugl Meyer (FM) is a reliable, valid, and responsive measure of motor function following stroke $[43,44]$. Its scores range between zero and 34 . The Berg balance scale (BBS) measures balance during functional tasks, and it consists of 14 items [45]. A five-point ordinal scale from zero to 4 is used to rate each item, in which a score of zero indicates poor balance and four indicates good balance ability. The RMI consists of 15 items (one of the items is direct observation of the patients, whereas the remaining 14 items are self-reports by the patients) arranged in increasing difficulty and scored on a scale of zero to one [46]. A score of zero indicates that the patient is unable to complete the task, whereas a score of one indicates that the patient is able to complete the task. The MAS is a reliable and valid scale that is rated on an ordinal scale of zero to four [47]. The 10MWT measures walking speed in meters per second over a short duration, and it has been reported to have excellent reliability for comfortable and fast gait speeds and predictive validity [48]. The 6MWT is also a valid and reliable submaximal exercise test for assessing aerobic capacity and endurance [49]. The hard activity chart is similarly a valid and reliable instrument [50]. All measurements were carried out at baseline, two weeks, and four weeks after the commencement of the interventions by blinded assessors.

The data obtained in the study was assessed for normality using the Kolmogorov-Smirnov statistics. Data for the demographic characteristics of the study participants was analyzed using descriptive statistics. The data on the study outcomes was analyzed using intention to treat analysis. However, in case of missing data, the mean imputation method was used [51]. Since the data obtained was not normally distributed $(p<0.05)$, change from baseline was analyzed using the Friedman test (for between-group data) and differences between groups were compared using the Mann-Whitney $U$ test. The level of significance was set at a level of significance of $<0.05$. Where there was a significant difference within group or change from baseline, the Wilcoxon signed-rank test was used for post hoc analysis. The level of significance for the multiple comparisons was set at $p<0.02$ using Bonferroni adjustment obtained by dividing 0.05 by three (the number of tests). All data were analyzed using SPSS version 20 . The study protocol was published earlier [52].

\section{Results}

A total of seventy-two people with stroke were assessed for the study in which only 58 (80.5\%) were eligible and participated in this study with age range, $18-75$ years (30 participants in group 1 and 28 in group 2). The reason why there were 30 participants in group 1 but only 28 in group 2 was because we wanted to keep the number of participants in each group equal but because there were two hospitals where the data was collected, this resulted in an equal number of participants in the two groups. However, the study compliance was $96.5 \%$; only two participants did not complete the study protocol (one had malaria and the other had no transport to the clinic). These participants were in group 2. In addition, only two participants (in group 1) reported mild low back pain and calf muscle pain, respectively. The characteristics of the study participants and the study flow chart are presented in Table 1 and Figure 1, respectively.

The results showed that, in both groups 1 and 2, there was significant improvement $(p<0.05)$ in the primary outcome (motor function) and the other study outcomes (balance, functional mobility, knee extensor spasticity, walking speed, endurance, and exertion before and after commencement of activities from baseline to 2 weeks and 4 weeks after the commencement of the intervention). Details of the results are presented in Table 2. For between-group comparisons, the results showed that there was only significant difference $(p<0.05)$ in knee extensor spasticity (group 1 (median $=$ $0(0)$, mean rank $=27.50)$; group 2 (median $=0(0)$, mean rank $=31.64)$ ), and exertion before commencement of activities (group $1($ median $=0(0.5)$, mean rank $=21.90)$; group 2 
TABLE 1: Baseline details on the study subjects.

\begin{tabular}{|c|c|c|}
\hline Variable & Group $1(n=30)$ & Group $2(n=28)$ \\
\hline \multicolumn{3}{|l|}{ Gender } \\
\hline Male & $12(48 \%)$ & $13(52 \%)$ \\
\hline Female & $18(44.6 \%)$ & $15(45.5 \%)$ \\
\hline Age (years) & $50.2 \pm 13.9$ & $47.8 \pm 14.7$ \\
\hline \multicolumn{3}{|l|}{ Stroke type } \\
\hline Ischemic & $25(83.3 \%)$ & $25(89.3 \%)$ \\
\hline Hemorrhagic & $5(16.7 \%)$ & $3(10.7 \%)$ \\
\hline \multicolumn{3}{|l|}{ Limb dominance } \\
\hline Right & $28(93.3 \%)$ & $22(78.6 \%)$ \\
\hline Left & $2(6.7 \%)$ & $6(21.4 \%)$ \\
\hline \multicolumn{3}{|l|}{ Side affected } \\
\hline Right & $20(66.7 \%)$ & $16(57.1 \%)$ \\
\hline Left & $10(33.3 \%)$ & $12(42.9 \%)$ \\
\hline Time since stroke (weeks) & $32(80.5)^{*}$ & $34(70)^{*}$ \\
\hline MMSE scores & $27(2)^{*}$ & $28(3)^{*}$ \\
\hline \multicolumn{3}{|l|}{ FAC scores } \\
\hline 2 & $12(40 \%)$ & $10(35.7 \%)$ \\
\hline 3 & $13(43.3 \%)$ & $14(50 \%)$ \\
\hline 4 & $5(16.7 \%)$ & $4(14.3 \%)$ \\
\hline SCT scores & $49(3.25)^{*}$ & $49(4.75)^{*}$ \\
\hline Knee flexion ROM (degrees) & $32.5^{\circ}(45)^{*}$ & $49.5^{\circ}(38.5)^{*}$ \\
\hline \multicolumn{3}{|l|}{ Vital signs } \\
\hline $\mathrm{RR}$ (cycles/min) & $17(5)^{*}$ & $18(4)^{*}$ \\
\hline PR (beats/min) & $89(14)^{*}$ & $82(23)^{*}$ \\
\hline SBP (mmHg) & $130 \pm 10.2$ & $126.4 \pm 15.3$ \\
\hline $\mathrm{DBP}(\mathrm{mmHg})$ & $80(12)^{*}$ & $85(16.5)^{*}$ \\
\hline Motor impairment & $62.5(8)^{*}$ & $62(9.5)$ \\
\hline Balance & $48(5.3)^{*}$ & $48(5.5)^{*}$ \\
\hline Functional mobility & $12(2)^{*}$ & $12(1)^{*}$ \\
\hline \multicolumn{3}{|l|}{ Knee extensor spasticity } \\
\hline 0 & $11(36.7 \%)$ & $16(57.14 \%)$ \\
\hline 1 & $15(53.6 \%)$ & $2(7.14 \%)$ \\
\hline 2 & $4(13.3 \%)$ & $10(35.71 \%)$ \\
\hline Walking speed (m/s) & $0.69(0.59)^{*}$ & $0.54(0.58)^{*}$ \\
\hline Walking endurance (meters) & $141(66.75)^{*}$ & $134(110.63)$ \\
\hline \multicolumn{3}{|l|}{ Exertion } \\
\hline 0 & $8(26.67 \%)$ & $10(35.71 \%)$ \\
\hline 0.5 & $6(20)$ & $9(30 \%)$ \\
\hline 1 & $8(26.67 \%)$ & $7(25 \%)$ \\
\hline 2 & $8(26.67 \%)$ & $1(3.33 \%)$ \\
\hline 3 & $0(0 \%)$ & $1(3.33 \%)$ \\
\hline
\end{tabular}

* = median and interquartile range; $\mathrm{MMSE}=$ minimental state examination; $\mathrm{FAC}=$ functional ambulation category; $\mathrm{SCT}=$ star cancellation test; $\mathrm{ROM}=$ range of motion; $\mathrm{RR}=$ respiratory rate; $\mathrm{PR}=$ pulse rate; $\mathrm{SBP}=$ systolic blood pressure; $\mathrm{DBP}=$ diastolic blood pressure .

$($ median $=1(0.5)$, mean rank $=37.64)$ ), and after commencement of activities (group 1 (median $=1(1)$, mean rank $=20.07)$; group $2($ median $=1(0)$, mean rank $=39.61)$ postintervention in favour of group 1). This is because the lower the scores for knee extensor spasticity and exertion, the better the improvement. Consequently, the mean ranks for group 1 are lower than those for group 2 in both knee extensor spasticity and exertion before and after commencement of activities. Details of the results are shown in Table 3.

\section{Discussion}

The aim of this study was to find out how effective is a lower limb CIMT protocol that uses number of repetitions of practice as a measure of intensity compared to a CIMT protocol that uses number of hours of practice at improving lower limb functional outcomes in people with stroke. The whole aim was to make therapy more efficient, make instructions to patients more specific, and try to increase therapy adherence by choosing one protocol. This is because the protocol using number of repetitions of practice seems to be easy to follow, specific, and efficient in upper limb CIMT $[3,36]$. When a protocol is specific and easy to follow, compliance and adherence with the protocol are better achieved. Consequently, all the participants in the number of repetitions of the practice group completed the study, indicating that the protocol may be more feasible. In addition, only two participants (in group 1) reported mild low back pain and calf muscle pain, respectively, indicating that the protocol is safe. Serious adverse events can raise safety concerns and restrict the utility of an intervention. However, it is not significant in this case considering the mild nature of the pain and that only $6.7 \%$ of the participants in the group reported just a few adverse events.

Similarly, the results show that there is no significant difference between the two protocols in motor function, balance, functional mobility, walking speed, and walking endurance. Previous studies in upper limb CIMT using a protocol that uses number of repetitions of practice as a measure of intensity of practice reported it to be both feasible and effective at improving motor outcomes such as real-world arm use and motor function [32-34, 36]. In addition, the protocol takes less time (about one hour) to complete compared to the traditional or modified CIMT in which several hours are used for practice with no information on the intensity of tasks practiced. Thus, the protocol that uses number of repetitions of practice as a measure of intensity of practice can be used as an effective alternative for the one that uses number of hours of task practice as a measure of intensity of practice.

Studies using number of hours of practice in their protocols are thought to be not clear on the amount of the tasks practiced [27]. This is probably because of several reasons. One, in contrast with logic, short duration of task practice ( $<3$ hours per day) has been shown to be more effective than long duration ( $\geq 3$ hours per day) of task practice. Secondly, there is no certainty on whether the number of hours claimed are completely used for task practice. Report and analysis of previous studies indicated that only 3.95 hours out of 6 hours and $33 \%$ of the total time, respectively, were used for practice $[53,54]$. However, the number of times tasks are practiced determines functional recovery [55], and in stroke literature, 


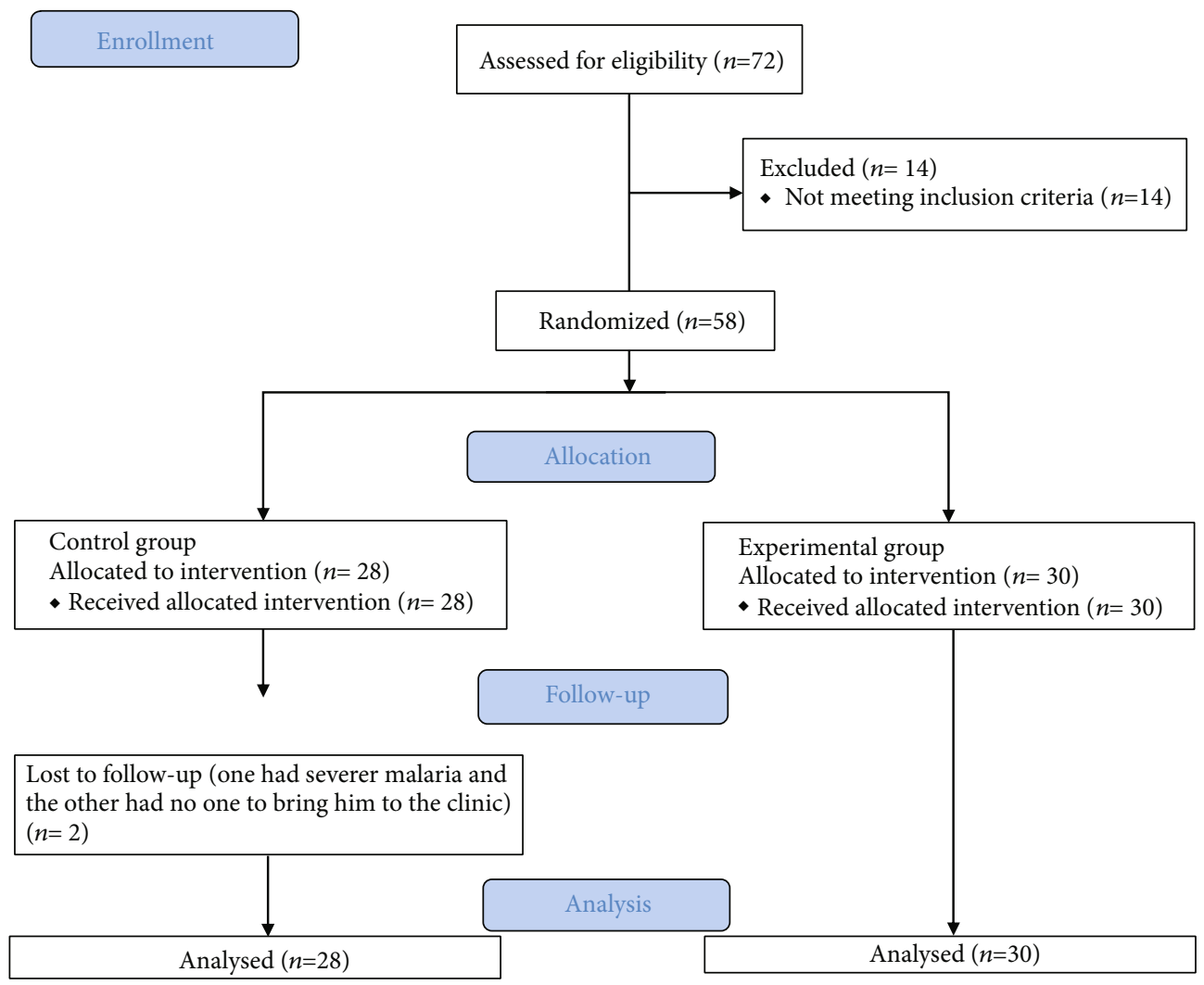

Figure 1: Study flowchart.

TABLE 2: Within group differences in the study outcomes.

\begin{tabular}{lcccccccccc}
\hline \multirow{2}{*}{ Variable } & \multicolumn{4}{c}{ Group $1(n=30)$} & \multicolumn{4}{c}{ Group 2(n=28) } & \multicolumn{3}{c}{ 2 } \\
& Baseline & 2 weeks & 4 weeks & $\lambda^{2}$ & $p$ value & Baseline & 2 weeks & 4 weeks & $\lambda^{2}$ & $p$ value \\
\hline Motor impairment & $62.5(8)$ & $63(3)$ & $64(7)$ & 50.634 & $<0.001$ & $62(10)$ & $63(9)$ & $64(8)$ & 39.000 & $<0.001$ \\
Balance & $48(5)$ & $50(5)$ & $51(4)$ & 51.869 & $<0.001$ & $48(6)$ & $49(7)$ & $50(7)$ & $50.060<0.001$ \\
Functional mobility & $12(2)$ & $13(1)$ & $13(2)$ & 36.324 & $<0.001$ & $12(1)$ & $13(0)$ & $13(0)$ & 29.778 & $<0.001$ \\
Knee extensor spasticity & $1(1)$ & $0(0)$ & $0(0)$ & 32.500 & $<0.001$ & $0(2)$ & $0(1)$ & $0(0)$ & $20.421<0.001$ \\
Walking speed & $0.69(0.59)$ & $0.63(0.74)$ & $0.66(0.87)$ & 41.862 & $<0.001$ & $0.54(0.57)$ & $0.60(0.64)$ & $0.66(0.71)$ & $30.500<0.001$ \\
Walking endurance & $141(66)$ & $169(90)$ & $205(117)$ & 27.748 & $<0.001$ & $134(111)$ & $138(126)$ & $226(115)$ & $40.775<0.001$ \\
Exertion 1 & $1(2)$ & $1(0.5)$ & $0(0.5)$ & 32.109 & $<0.001$ & $0.5(1)$ & $0.5(0.5)$ & $1(0.5)$ & 7.253 & 0.027 \\
Exertion 2 & $2(1)$ & $2(2)$ & $1(1)$ & 24.930 & $<0.001$ & $2(2)$ & $2(1)$ & $2(0)$ & 10.167 & 0.006 \\
\hline
\end{tabular}

Exertion 1 = exertion before commencement of activities; exertion $2=$ exertion after commencement of activities.

this number of repetitions has been determined [29-33]. According to Abdullahi, this number of repetitions can be achieved within one hour [27]. One hour of practice is far less than the time used in the traditional CIMT and many modified protocols of CIMT. This seems to suggest that it is not the time spent that matters, but how many times tasks are practiced. Thus, a CIMT protocol using number of repetitions of practice seems more desirable and easier to practice, whereas the CIMT protocol using number of hours of practice is time consuming. To the best of our knowledge, this study seems to be the only fully fledged RCT comparing a lower limb CIMT protocol that uses number of repetitions of practice with the one that uses number of hours of practice.
Furthermore, in the present study, knee extensor spasticity and exertion before and after the commencement of activity reduced more in group 1 than in group 2 at four weeks postintervention. Similarly, at two weeks postintervention, the reduction in exertion after commencement of activity has borderline significance in favour of group 1. This is an important finding since exertion can hinder task practice and delay walking recovery, as energy expenditure and cost during walking tend to be high in stroke patients [56]. However, according to Billinger and colleagues, consistent single limb exercise is an effective method for improving oxygen uptake and reducing energy expenditure during submaximal effort [21]. Therefore, the reduction in exertion in the present study which is better in group 1 could be explained by the 
TABLE 3: Between-group differences in the study outcomes.

\begin{tabular}{|c|c|c|c|c|c|}
\hline Time period & Variable & Group $1(n=30)$ & Group $1(n=28)$ & $U$ & $p$ value \\
\hline \multirow{8}{*}{ Baseline } & Motor impairment & $62.59(8)$ & $62(10)$ & 356.000 & 0.321 \\
\hline & Balance & $48(5)$ & $48(6)$ & 405.000 & 0.820 \\
\hline & Functional mobility & $12(2)$ & $12(1)$ & 366.000 & 0.386 \\
\hline & Knee extensor spasticity & $1(1)$ & $0(2)$ & 405.000 & 0.801 \\
\hline & Walking speed & $0.69(0.59)$ & $0.54(0.57)$ & 417.000 & 0.963 \\
\hline & Walking endurance & $141(66)$ & $134(111)$ & 385.000 & 0.586 \\
\hline & Exertion 1 & $1(2)$ & $0.5(1)$ & 321.000 & 0.111 \\
\hline & Exertion 2 & $2(1)$ & $2(2)$ & 347.000 & 0.236 \\
\hline \multirow{8}{*}{2 weeks } & Motor impairment & $63(7)$ & $63(9)$ & 332.000 & 0.170 \\
\hline & Balance & $50(5)$ & $49(7)$ & 370.500 & 0.438 \\
\hline & Functional mobility & $13(1)$ & $13(0)$ & 397.000 & 0.698 \\
\hline & Knee extensor spasticity & $0(0)$ & $0(1)$ & 387.000 & 0.450 \\
\hline & Walking speed & $0.63(0.74)$ & $0.61(0.64)$ & 416.000 & 0.950 \\
\hline & Walking endurance & $169(90)$ & $138(126)$ & 377.000 & 0.503 \\
\hline & Exertion 1 & $1(0.5)$ & $0.5(0.5)$ & 337.500 & 0.049 \\
\hline & Exertion 2 & $2(2)$ & $2(1)$ & 300.000 & 0.049 \\
\hline \multirow{8}{*}{4 weeks } & Motor impairment & $64(7)$ & $64(8)$ & 331.000 & 0.166 \\
\hline & Balance & $51(4)$ & $50(7)$ & 417.000 & 0.963 \\
\hline & Functional mobility & $13(2)$ & $13(0)$ & 350.000 & 0.208 \\
\hline & Knee extensor spasticity & $0(0)$ & $0(0)$ & 384.000 & 0.033 \\
\hline & Walking speed & $0.66(0.87)$ & $0.66(0.62)$ & 406.500 & 0.834 \\
\hline & Walking endurance & $205(117)$ & $226(115)$ & 303.500 & 0.070 \\
\hline & Exertion 1 & $0(0.5)$ & $1(0.5)$ & 137.000 & $<0.001$ \\
\hline & Exertion 2 & $1(1)$ & $1(0)$ & 192.000 & $<0.001$ \\
\hline
\end{tabular}

Exertion 1 = exertion before commencement of activities; exertion 2 = exertion after commencement of activities.

virtue of the consistency in the exercise performance since participants had to practice the same number of repetitions of practice every day. Similarly, reduced spasticity in group 1 was noted in the knee extensors. Knee extensor spasticity is one of the sequelae of stroke, and improving this may be an important stroke rehabilitation milestone as it may help patients achieve independence in walking and other daily activities just like reduced exertion. Previous studies however showed that spasticity does not significantly contribute to walking dysfunction after stroke [57-59], but knee extensor muscles torque does [57]. The problems with these studies are that the sample size was small and those with spasticity were too small in number, respectively. Additionally, correlation does not mean cause and effect. In contrast, in the present study, spasticity and walking ability significantly improved after the intervention. This seems to suggest that spasticity may negatively affect walking ability and independence in patients with stroke.

Another issue in the study is feasibility of the group 1 protocol which was high, and interestingly, there were only a few adverse effects reported. In contrast, analysis of CIMT studies using number of hours of practice revealed that the compliance was not optimal $[53,54]$. Although the authors could not give reasons for the nonoptimal compliance, this may be because of the demand of the protocol in terms of supervision, length of time, and lack of accountability in recording the intensity of practice. Thus, CIMT using number of task repetition may be a better alternative since task repetition can be counted even by the patients themselves [60]. However, the present study has some limitations too. One of the limitations is the majority of the participants $(65 \%)$ in the study were in chronic stage of stroke $(>6$ months), and as such, the improvement in the outcomes of interest such as walking distance, functional ability, and balance might be as a result of compensation. Recovery of motor function is usually slow and small after 6 months poststroke [61-63]. Additionally, CIMT studies in chronic stroke patients usually combine CIMT with brain stimulation in order to achieve any improvement $[64,65]$. Secondly, the time taken to perform the number of repetitions per session was not recorded, although previously, it was shown that 300 repetitions of task practice were possible within one hour [32]. In conclusion, the protocol of lower limb CIMT using number of repetitions of task practice is feasible and equally as effective as the one using number to hours of task practice. Therefore, number of repetitions of practice can serve as a suitable alternative of number of hours of practice since the intensity of task practice required for recovery of motor function is known when the former is used. 


\section{Data Availability}

The data for the study is available on request to the corresponding author.

\section{Conflicts of Interest}

The authors declare no conflict of interest.

\section{Acknowledgments}

The authors would like to thank the study participants for participating in the study. Similarly, we would like to thank the staff at the study sites who helped in the process of conducting the research.

\section{Supplementary Materials}

CONSORT 2010 checklist of information to include when reporting a randomized trial*. (Supplementary Materials)

\section{References}

[1] R. A. Schmidt, "A schema theory of discrete motor skill learning," Psychological Review, vol. 82, no. 4, pp. 225-260, 1975.

[2] J. M. Hollerbach, "Computers, brains and the control of movement," Trends in Neurosciences, vol. 5, no. 6, pp. 189-192, 1982.

[3] J. F. Yang and M. Gorassini, "Spinal and brain control of human walking: implications for retraining of walking," The Neuroscientist, vol. 12, no. 5, pp. 379-389, 2006.

[4] H. S. Jorgensen, H. Nakayama, H. O. Raaschou, J. Vive-Larsen, M. Stoier, and T. S. Olsen, "Outcome and time course of recovery in stroke. Part II: time course of recovery. The Copenhagen Stroke Study," Archives of Physical Medicine and Rehabilitation, vol. 76, no. 5, pp. 406-412, 1995.

[5] M. A. Gatti, M. Portela, M. Gianella et al., "Walking ability after stroke in patients from Argentina: predictive values of two tests in subjects with subacute hemiplegia," Journal of Physical Therapy Science, vol. 27, no. 9, pp. 2977-2980, 2015.

[6] G. G. Hirschberg, "Ambulation and self-care are goals of rehabilitation after stroke," Geriatrics, vol. 31, no. 5, pp. 61-65, 1976.

[7] C. Bonnyaud, D. Pradon, R. Zory et al., "Effects of a gait training session combined with a mass on the non-paretic lower limb on locomotion of hemiparetic patients: a randomized controlled clinical trial," Gait \& Posture, vol. 37, no. 4, pp. 627-630, 2013.

[8] Q. Ding, I. H. Stevenson, N. Wang et al., "Motion games improve balance control in stroke survivors: a preliminary study based on the principle of constraint-induced movement therapy," Displays, vol. 34, no. 2, pp. 125-131, 2013.

[9] E. Taub and A. J. Berman, "Avoidance conditioning in the absence of relevant proprioceptive and exteroceptive feed back," Journal of Comparative and Physiological Psychology, vol. 56, no. 6, pp. 1012-1016, 1963

[10] I. Marklund and M. Klässbo, "Effects of lower limb intensive mass practice in poststroke patients: single-subject experimental design with long-term follow-up," Clinical Rehabilitation, vol. 20 , no. 7 , pp. 568-576, 2016.
[11] K. Kallio, L. Nilsson-Wikmar, and A. M. Thorsén, "Modified constraint-induced therapy for the lower extremity in elderly persons with chronic stroke: single-subject experimental design study," Topics in Stroke Rehabilitation, vol. 21, no. 2, pp. 111-119, 2014.

[12] E. M. G. d. S. e Silva, T. S. Ribeiro, T. C. C. da Silva, M. F. P. Costa, F. A. d. C. Cavalcanti, and A. R. R. Lindquist, "Effects of constraint-induced movement therapy for lower limbs on measurements of functional mobility and postural balance in subjects with stroke: a randomized controlled trial," Topics in Stroke Rehabilitation, vol. 24, no. 8, pp. 555-561, 2017.

[13] C. G. Ostendorf and S. L. Wolf, "Effect of forced use of the upper extremity of a hemiplegic patient on changes in function. A single-case design," Physical Therapy, vol. 61, no. 7, pp. 1022-1028, 1981.

[14] E. Taub, N. E. Miller, T. A. Novack et al., "Technique to improve chronic motor deficit after stroke," Archives of Physical Medicine and Rehabilitation, vol. 74, no. 4, pp. 347-354, 1993.

[15] S. L. Wolf, C. J. Winstein, J. P. Miller et al., "Effect of constraint-induced movement therapy on upper extremity function 3 to 9 months after stroke," JAMA, vol. 296, no. 17, pp. 2095-2104, 2006.

[16] A. S. Aruin, N. Rao, A. Sharma, and G. Chaudhuri, "Compelled body weight shift approach in rehabilitation of individuals with chronic stroke," Topics in Stroke Rehabilitation, vol. 19, no. 6, pp. 556-563, 2014.

[17] H.-S. Choi, W.-S. Shin, D.-H. Bang, and S.-J. Choi, "Effects of game-based constraint-induced movement therapy on balance in patients with stroke: a single-blind randomized controlled trial," American Journal of Physical Medicine \& Rehabilitation, vol. 96, no. 3, pp. 184-190, 2017.

[18] M. Etoom, M. Hawamdeh, Z. Hawamdeh et al., "Constraintinduced movement therapy as a rehabilitation intervention for upper extremity in stroke patients," International Journal of Rehabilitation Research, vol. 39, no. 3, pp. 197-210, 2016.

[19] M. A. Abba, A. S. Muhammad, U. M. Badaru, and A. Abdullahi, "Comparative effect of constraint-induced movement therapy and proprioceptive neuromuscular facilitation on upper limb function of chronic stroke survivors," Physiotherapy Quarterly, vol. 28, no. 1, pp. 1-5, 2020.

[20] A. Abdullahi, S. Truijen, and W. Saeys, "Neurobiology of recovery of motor function after stroke: the central nervous system biomarker effects of constraint-induced movement therapy," Neural Plasticity, vol. 2020, Article ID 9484298, 12 pages, 2020.

[21] S. A. Billinger, L. X. Guo, P. S. Pohl, and P. M. Kluding, "Single limb exercise: pilot study of physiological and functional responses to forced use of the hemiparetic lower extremity," Topics in Stroke Rehabilitation, vol. 17, no. 2, pp. 128-139, 2015.

[22] A. C. Fuzaro, C. T. Guerreiro, F. C. Galetti, R. B. V. M. Jucá, and J. E. de Araujo, "Modified constraint-induced movement therapy and modified forced-use therapy for stroke patients are both effective to promote balance and gait improvements," Rev Bras Fisioter, pp. 1-9, 2012.

[23] R. Adami, J. Pagano, M. Colombo et al., "Reduction of movement in neurological diseases: effects on neural stem cells characteristics," Frontiers in Neuroscience, vol. 12, p. 336, 2018.

[24] K. Angata and M. Fukuda, "Roles of polysialic acid in migration and differentiation of neural stem cells," Methods in Enzymology, vol. 479, no. 479, pp. 25-36, 2010. 
[25] L. A. Vearrier, J. Langan, A. Shumway-Cook, and M. Woollacott, "An intensive massed practice approach to retraining balance post-stroke," Gait \& Posture, vol. 22, no. 2, pp. 154-163, 2005.

[26] S. dos Anjos, D. M. Morris, and E. Taub, "Constraint-induced movement therapy for improving motor function of the paretic lower extremity after stroke," American Journal of Physical Medicine \& Rehabilitation, vol. 99, no. 6, p. 1, 2020.

[27] A. Abdullahi, "Is time spent using constraint induced movement therapy an appropriate measure of dose? A critical literature review," International Journal of Therapy and Rehabilitation, vol. 21, no. 3, pp. 140-145, 2014.

[28] J. A. Kleim, S. Barbay, and R. J. Nudo, "Functional reorganization of the rat motor cortex following motor skill learning," Journal of Neurophysiology, vol. 80, no. 6, pp. 3321-3325, 1998.

[29] J. R. Carey, T. J. Kimberley, S. M. Lewis et al., "Analysis of fMRI and finger tracking training in subjects with chronic stroke," Brain, vol. 125, no. 4, pp. 773-788, 2002.

[30] L. Boyd and C. Winstein, "Explicit information interferes with implicit motor learning of both continuous and discrete movement tasks after stroke," Journal of Neurologic Physical Therapy, vol. 30, no. 2, pp. 46-57, 2006.

[31] M. S. Fine and K. A. Thoroughman, "Motor adaptation to single force pulses: sensitive to direction but insensitive to within movement pulse placement and magnitude," Journal of Neurophysiology, vol. 96, no. 2, pp. 710-720, 2006.

[32] R. L. Birkenmeier, E. M. Prager, and C. E. Lang, "Translating animal doses of task-specific training to people with chronic stroke in 1-hour therapy sessions: a proof-of-concept study," Neurorehabilitation and Neural Repair, vol. 24, no. 7, pp. 620-635, 2010.

[33] K. J. Waddell, R. L. Birkenmeier, J. L. Moore, T. G. Hornby, and C. E. Lang, "Feasibility of high-repetition, task-specific training for individuals with upper-extremity paresis," American Journal of Occupational Therapy, vol. 68, no. 4, pp. 444453, 2014.

[34] A. Abdullahi, S. Shehu, and I. B. Dantani, "Feasibility of high repetition of task practice in constraint induced movement therapy in an acute stroke patient," International Journal of Therapy and Rehabilitation, vol. 21, no. 4, pp. 190-195, 2014.

[35] J. Schröder, S. Truijen, T. Van Criekinge, and W. Saeys, "Feasibility and effectiveness of repetitive gait training early after stroke: a systematic review and meta-analysis," Journal of Rehabilitation Medicine, vol. 51, no. 2, pp. 78-88, 2019.

[36] A. Abdullahi, "Effects of number of repetitions and number of hours of shaping practice during constraint-induced movement therapy: a randomized controlled trial," Neurology Research International, vol. 2018, Article ID 5496408, 9 pages, 2018.

[37] F. Faul, E. Erdfelder, A. G. Lang, and A. Buchner, "G * Power 3 : a flexible statistical power analysis program for the social, behavioral, and biomedical sciences," Behavior Research Methods, vol. 39, no. 2, pp. 175-191, 2007.

[38] K. A. Danlami and A. Abdullahi, "Remodelling the protocol of lower limb constraint induced movement therapy: a randomized controlled trial," Arch Physiother Global Res, vol. 21, no. 4, pp. 21-27, 2017.

[39] S. Brunnstrom, Movement Therapy in Hemiplegia: A Neurophysiological Approach, Harper \& Row, New York, NY, 1970.

[40] Stroke engine, "Star cancellation test," November 2019, https://www.strokengine.ca/en/quick/sct_quick/.
[41] S. J. Page, P. Levine, A. Leonard, J. P. Szaflarski, and B. M. Kissela, "Modified constraint-induced therapy in chronic stroke: results of a single-blinded randomized controlled trial," Physical Therapy, vol. 88, no. 3, pp. 333-340, 2008.

[42] Cleveland Clinic, "Rated Perceived Exertion Scale' by Cleveland Clinic," 2019, https://my.clevelandclinic.org/health/ articles/17450-rated-perceived-exertion-rpe-scale.

[43] A. R. Fugl-Meyer, L. Jääskö, I. Leyman, S. Olsson, and S. Steglind, "The post-stroke hemiplegic patient. 1. A method for evaluation of physical performance," Scandinavian Journal of Rehabilitation Medicine, vol. 7, no. 1, pp. 13-31, 1975.

[44] D. J. Gladstone, C. J. Danells, and S. E. Black, “The Fugl-Meyer assessment of motor recovery after stroke: a critical review of its measurement properties," Neurorehabilitation and Neural Repair, vol. 16, no. 3, pp. 232-240, 2002.

[45] K. Berg, S. Wood-Dauphinee, J. I. Williams, and B. Maki, "Measuring balance in the elderly: validation of an instrument," Canadian Journal of Public Health, vol. 2, pp. 7-11, 1992.

[46] H. M. Chen, C. L. Hsieh, Sing Kai Lo, L. J. Liaw, S. M. Chen, and J. H. Lin, "The test-retest reliability of 2 mobility performance tests in patients with chronic stroke," Neurorehabilitation and Neural Repair, vol. 21, no. 4, pp. 347-352, 2007.

[47] N. Ghotbi, N. N. Ansari, S. Naghdi, S. Hasson, B. Jamshidpour, and S. Amiri, "Inter-rater reliability of the modified modified Ashworth scale in assessing lower limb muscle spasticity," Brain Injury, vol. 23, no. 10, pp. 815-819, 2009.

[48] S. Tyson and L. Connell, "The psychometric properties and clinical utility of measures of walking and mobility in neurological conditions: a systematic review," Clinical Rehabilitation, vol. 23, no. 11, pp. 1018-1033, 2009.

[49] P. Nogueira, A. Leal, C. Pulz, I. Nogueira, and J. Filho, "Clinical reliability of the 6 minute corridor walk test performed within a week of a myocardial infarction," International Heart Journal, vol. 47, no. 4, pp. 533-540, 2006.

[50] L. Herman, C. Foster, M. A. Maher, R. P. Mikat, and J. P. Porcari, "Validity and reliability of the session RPE method for monitoring exercise training intensity," South African Journal of Sports Medicine, vol. 18, no. 1, pp. 14-17, 2006.

[51] Columbia University, "Missing-data imputatio, Chapter 25: Columbia Statistics," November 2018, http://www.stat .columbia.edu/_gelman/arm/missing.pdf.

[52] N. A. Umar and A. Abdullahi, "Comparison between two different protocols of lower limb constraint-induced movement therapy following stroke: a randomised controlled trial protocol," European Journal of Physiotherapy, vol. 22, no. 3, pp. 178-182, 2019.

[53] R. T. Kaplon, M. G. Prettyman, C. L. C. J. Kushi, and C. J. Winstein, "Six hours in the laboratory: a quantification of practice time during constraint-induced therapy (CIT)," Clinical Rehabilitation, vol. 21, no. 10, pp. 950-958, 2016.

[54] R. Stock, G. Thrane, T. Askim et al., "Norwegian constraintinduced therapy multisite trial: adherence to treatment protocol applied early after stroke," Journal of Rehabilitation Medicine, vol. 47, no. 9, pp. 816-823, 2015.

[55] R. J. Nudo and G. W. Milliken, "Reorganization of movement representations in primary motor cortex following focal ischemic infarcts in adult squirrel monkeys," Journal of Neurophysiology, vol. 75, no. 5, pp. 2144-2149, 1996.

[56] S. Kramer, L. Johnson, J. Bernhardt, and T. Cumming, "Energy expenditure and cost during walking after stroke: a systematic 
review," Archives of Physical Medicine and Rehabilitation, vol. 97, no. 4, pp. 619-632.e1, 2016.

[57] R. W. Bohannon and A. W. Andrews, "Correlation of knee extensor muscle torque and spasticity with gait speed in patients with stroke," Archives of Physical Medicine and Rehabilitation, vol. 71, no. 5, pp. 330-333, 1990.

[58] L. Ada, W. Vattanasilp, N. J. O'Dwyer, and J. Crosbie, "Does spasticity contribute to walking dysfunction after stroke?," Journal of Neurology, Neurosurgery \& Psychiatry, vol. 64, no. 5, pp. 628-635, 1998.

[59] D. K. Sommerfeld, E. U.-B. Eek, A. K. Svensson, L. W.. Holmqvist, and M. H. von Arbin, "Spasticity after stroke its occurrence and association with motor impairments and activity limitations," Stroke, vol. 35, no. 1, pp. 134-139, 2004.

[60] P. Bagley, M. Hudson, J. Green, A. Forster, and J. Young, "Do physiotherapy staff record treatment time accurately? An observational study," Clinical Rehabilitation, vol. 23, no. 9, pp. 841-845, 2009.

[61] P. J. Friedman, "Gait recovery after hemiplegic stroke," International Disability Studies, vol. 12, no. 3, pp. 119-122, 2009.

[62] P. W. Duncan and S. Min Lai, "Stroke recovery," Topics in Stroke Rehabilitation, vol. 4, no. 3, pp. 51-58, 2015.

[63] J. H. Cauraugh and J. J. Summers, "Neural plasticity and bilateral movements: a rehabilitation approach for chronic stroke," Progress in Neurobiology, vol. 75, no. 5, pp. 309-320, 2005.

[64] C. Y. Wu, Y. W. Hsieh, K. C. Lin et al., "Brain reorganization after bilateral arm training and distributed constraintinduced therapy in stroke patients: a preliminary functional magnetic resonance imaging study," Chang Gung Medical Journal, vol. 33, no. 6, pp. 628-638, 2010.

[65] N. Bolognini, G. Vallar, C. Casati et al., "Neurophysiological and behavioral effects of tDCS combined with constraintinduced movement therapy in poststroke patients," Neurorehabilitation and Neural Repair, vol. 25, no. 9, pp. 819-829, 2011. 